\title{
Cerebrospinal Fluid Analysis Predicts Enhancement on MRI in Bacterial and Tuberculous Meningitis: A Retrospective Observational Study
}

Ryoji Goto ( $\sim$ gotoryoji59@gmail.com )

National Center for Global Health and Medicine: Kokuritsu Kenkyu Kaihatsu Hojin Kokuritsu Kokusai Iryo Kenkyu Center https://orcid.org/0000-0002-1693-9915

\section{Yurino Horiuchi}

National Center for Global Health and Medicine: Kokuritsu Kenkyu Kaihatsu Hojin Kokuritsu Kokusai Iryo Kenkyu Center

\section{Haruka Kawakami}

National Center for Global Health and Medicine: Kokuritsu Kenkyu Kaihatsu Hojin Kokuritsu Kokusai Iryo Kenkyu Center

\section{Ayaka Chikada}

National Center for Global Health and Medicine: Kokuritsu Kenkyu Kaihatsu Hojin Kokuritsu Kokusai Iryo Kenkyu Center

\section{Tsutomu Yasuda}

National Center for Global Health and Medicine: Kokuritsu Kenkyu Kaihatsu Hojin Kokuritsu Kokusai Iryo Kenkyu Center

\section{Sousuke Takeuchi}

National Center for Global Health and Medicine: Kokuritsu Kenkyu Kaihatsu Hojin Kokuritsu Kokusai Iryo Kenkyu Center

\section{Noritoshi Arai}

National Center for Global Health and Medicine: Kokuritsu Kenkyu Kaihatsu Hojin Kokuritsu Kokusai Iryo Kenkyu Center

\section{Research}

Keywords: Meningitis, Bacterial meningitis, Tuberculous meningitis, Cerebrospinal fluid, Blood-brain barrier

Posted Date: April 14th, 2021

DOI: https://doi.org/10.21203/rs.3.rs-408740/v1 
License: (c) (i) This work is licensed under a Creative Commons Attribution 4.0 International License. Read Full License 


\section{Abstract}

\section{Background}

Along with cerebrospinal fluid (CSF) analysis, enhancement on contrast-enhanced MRI scans are useful to diagnose and presume the pathogen of meningitis. However, the conditions for its appearance have not been clarified. This study aimed to investigate the possibility of CSF parameters as predictors of the existence of enhancement on contrast-enhanced head or spinal MRI scans in patients with bacterial meningitis (BM) or tuberculous meningitis (TM).

Methods

A total of 12 patients with BM and 23 patients with TM who underwent both CSF analyses and contrastenhanced MRI scans were included in this study. The correlation between CSF analyses and MRI findings has been examined using receiver operating characteristic (ROC) analysis.

Results

Contrast enhancement was found in 7 and 10 patients with BM and TM, respectively. In patients with BM, higher CSF protein and lower CSF glucose were associated with the presence of the enhancement on MRI, while the CSF leukocyte or neutrocyte count did not show any difference. In contrast, not only the CSF protein and glucose but also the leukocyte and lymphocyte counts were associated with the enhancement in patients with TM. Furthermore, CSF adenosine deaminase (ADA) in patients with TM showed neither correlation with CSF leukocyte count nor discriminant ability of the MRI findings.

\section{Conclusions}

CSF analysis predicts the existence of enhancement on contrast-enhanced MRI scans of the central nervous system both in patients with BM and those with TM. Our findings about the CSF cell count and CSF ADA indicate the mechanism of the blood-brain barrier (BBB) breakdown in BM and TM.

\section{Background}

Bacterial meningitis (BM) and tuberculous meningitis (TM) are both rare but known to have high mortality rates and serious sequelae. While cerebrospinal fluid (CSF) analysis is useful to diagnose and presume the pathogen of meningitis, head and spinal MRI scans are also often effective not only in the diagnosis of meningitis but also in searching for complications. Enhancement, especially of the leptomeninges, on post-contrast MRI is known to be specific ${ }^{1,2}$, which is assumed to reflect the bloodbrain barrier (BBB) breakdown ${ }^{3}$.

However, abnormal enhancement is seen only in half of the patients, and the conditions for its appearance have not been clarified ${ }^{4}$. In this study, the possibility of CSF parameters as predictors of the existence of enhancement on contrast-enhanced MRI scans, both in patients with BM and those with TM, 
has been examined. The receiver operating characteristic (ROC) curves for the ability of each parameter to estimate the appearance of abnormal enhancement were plotted. The area under the curve (AUC) was calculated in each parameter. In addition, the data of those with BM and those with TM have been compared to discuss the mechanism of BBB breakdown.

\section{Methods}

Patients In this case series, adult patients (18 years old or more) who were diagnosed and treated as having BM or TM, underwent both CSF analyses and contrast-enhanced MRI of the central nervous system, and had been admitted to our institutions for meningitis between January 2011 and February 2021 have been recruited. As for BM, patients were eligible if they had been suspected with or proven to have BM by either (1) positive CSF culture or (2) CSF leukocyte count of more than $100 / \mu \mathrm{L}$ with symptoms and signs of acute BM, including headache, neck stiffness, fever, and systemic signs of infections, in addition to improvement after antibiotic treatment. As for TM, patients with definite, probable, or possible TM of the established diagnostic criteria 5 were eligible. Clinical data, including age, sex, past medical history, presenting symptoms, clinical course, duration of hospitalization, medication, and neurologic status at discharge, were collected in all patients. Sequelae in patients were defined as complications that resulted from meningitis present at the time of discharge. Sequelae included hearing loss, vision loss, cognitive dysfunction, aphasia, motor impairment, dysuria, and epilepsy. Routine blood test included leukocyte count and C-reactive protein (CRP). Routine CSF analyses included leukocyte count and its fractionations, CSF protein concentration (missing for 1), CSF glucose, and CSF culture. A few patients with TM lacked CSF adenosine deaminase (ADA). Since whether blood glucose was collected at the same time with CSF test or not was unclear in some cases, the analysis of CSF/serum glucose ratio was not performed. The duration of antibiotic administration was analyzed only in patients with BM, while CSF ADA was analyzed only in patients with TM. In addition, CSF protein concentration and CSF leukocyte count were log10-transformed before statistical analysis because of those skewed distributions. MRI studies were mostly performed on either a 3-Tesla MRI system (MAGNETOM Verio, Siemens Healthcare, Erlangen, Germany) or 1.5-Tesla MRI systems (MAGNETOM Aera and/or MAGNETOM Avanto, Siemens Healthcare, Erlangen, Germany; EXCELART Vantage Atlas, Canon Medical Systems, Otawara-shi, Japan; or Signa Excite HD and/or Signa HDxt, GE Healthcare, Milwaukee, WI, USA) using a standardized protocol including contrast-enhanced T1-weighted sequences. A 0.3-Tesla open MRI system (AIRIS Vento, Hitachi Healthcare, Kashiwa-shi, Japan) was performed using the same protocol in only one case because of claustrophobia of the patient. All MRI data, including those taken at the hospital where patients were admitted prior to transfer, were analyzed to detect new enhancement, with a structured reporting scheme independently by two reviewers unaware of the clinical detail and experienced with diagnostic and research studies using contrast-enhanced T1-weighted MRI. While head MRI scans were taken and analyzed in all patients, spinal MRI data were also taken in three patients with BM initially. Those blood, CSF, and MRI findings obtained just before treatment for meningitis were selected or, if obtained after starting the treatment, the initial test results. Among the patients who received inpatient treatment at their previous institution, the findings there were used to minimize the 
impact of therapeutic intervention. Statistics Categorical and continuous variables were compared using the $\chi 2$ test and the paired or unpaired Student's t-test, as appropriate. Spearman's rank correlation coefficient was used to verify the correlation. The overall accuracy of the potential variables in predicting the existence of enhancement on MRI was estimated by calculating the AUC and its standard error of ROC curves using a nonparametric method6. The optimal cutoff points of each parameter to predict the existence of enhancement on MRI were determined using the Youden's index, i.e., $\mathrm{J}=$ max (sensitivity + specificity-1)7. The significance of differences between areas under curves was also determined using the DeLong method. A p value $<0.05$ was considered statistically significant. All statistical analyses were performed using R version 3.6.3 (R Foundation for Statistical Computing, Vienna, Austria).

\section{Results}

\section{Patients and samples}

A total of 12 patients with BM and 24 patients with TM fulfilled the inclusion criteria. One patient with TM was excluded from the analysis because of having been treated at a hospital abroad; the medical record of this patient could not be accessed. The CSF protein in 34 patients (11 patients with BM and all of the patients with TM) has been analyzed, with the CSF ADA in 19 patients with TM. While blood neutrocyte and lymphocyte counts were analyzed in all patients with TM, they were analyzed only in seven patients with BM (5 with enhancement and 2 without).

On their initial MRI, contrast enhancement was detected in 7 out of 12 patients with BM and 9 out of 23 patients with TM. Among those with BM, four cases showed pia-subarachnoid space enhancement, which is known to be a typical finding in BM. Moreover, a dura-arachnoid space enhancement was found in two cases, while one patient showed cortical enhancement in suspected laminar necrosis. Another one patient showed enhancement only on spinal MRI. Among those with TM, all cases showed piasubarachnoid space enhancement on head MRI, especially in the basal cisterns and sylvian fissure. Five patients also showed intracranial tuberculomas and their enhancement. Furthermore, six patients developed hydrocephalus as a sequela during their clinical courses. Examples of contrast enhancement on MRI in patients with BM and those with TM are shown in Figure 1.

The whole characteristics of our cases (12 BM cases and 23 TM cases) are shown in Tables 1 and 2 . As for patient background, those with enhancement in patients with TM were younger $(p=0.045)$. Other characteristics did not show any differences. Some of the CSF or blood parameters clearly differed between the patients with and without new enhancement among those with TM. Compared with the patients without enhancement, CSF protein, leukocyte, lymphocyte, and blood leukocyte counts were higher $(p<0.05)$ in those with enhancement, while CSF glucose value was lower $(p=0.005)$. The additional data file shows their profile in more detail [see Additional file 1].

\section{Table 1. Characteristics of patients with bacterial meningitis}




\begin{tabular}{|llll|}
\hline & $\begin{array}{l}\text { With new } \\
\text { enhancement } \\
(\mathrm{n}=7)\end{array}$ & $\begin{array}{l}\text { Without new } \\
\text { enhancement } \\
(\mathrm{n}=5)\end{array}$ & $\begin{array}{c}p \\
\text { value }\end{array}$ \\
\hline Age & $57.7 \pm 15.3$ & $58.2 \pm 16.1$ & 0.962 \\
\hline No (\%) of women & $2(29)$ & $1(20)$ & 0.079 \\
\hline Underlying condition & $4(57)$ & $1(20)$ & 0.163 \\
\hline Blood leukocytes, 10^3/ $\mu \mathrm{L}$ & $17.2 \pm 4.84$ & $15.5 \pm 5.21$ & 0.608 \\
\hline Blood CRP, mg/dL & $14.2 \pm 9.87$ & $15.7 \pm 16.9$ & 0.867 \\
\hline CSF glucose, $\mathrm{mg} / \mathrm{dL}$ & $13 \pm 19$ & $73 \pm 77$ & 0.098 \\
\hline CSF protein, $\mathrm{mg} / \mathrm{dL}$ & $523(47-1672)$ & $201(28-255)$ & 0.117 \\
\hline CSF leukocytes, cells/ $\mu \mathrm{L}$ & $535(3.4-25327)$ & $1276(9-6213)$ & 0.989 \\
\hline CSF neutrocytes, cells/ $\mu \mathrm{L}$ & $529(0.7-22848)$ & $472(7-5643)$ & 0.991 \\
\hline CSF lymphocytes, cells/ $\mu \mathrm{L}$ & $13(3-2479)$ & $523(2-804)$ & 0.711 \\
\hline $\begin{array}{l}\text { Duration of antibiotic administration, } \\
\text { days }\end{array}$ & $49.7 \pm 24.2$ & $24.4 \pm 3.1$ & 0.060 \\
\hline No (\%) of patients with sequela & $2(29)$ & $4(80)$ & 0.079 \\
\hline
\end{tabular}

Abbreviations: CRP, C reactive protein; CSF, cerebrospinal fluid Table 2. Characteristics of patients with tuberculous meningitis 


\begin{tabular}{|c|c|c|c|}
\hline & $\begin{array}{l}\text { With new enhancement } \\
(n=10)\end{array}$ & $\begin{array}{l}\text { Without new enhancement } \\
(n=13)\end{array}$ & $p$ value \\
\hline Age & $45.8 \pm 19.0$ & $65.5 \pm 22.4$ & 0.045 \\
\hline No (\%) of women & $5(50)$ & $5(38)$ & 0.405 \\
\hline Underlying condition & $3(30)$ & $7(54)$ & 0.195 \\
\hline Blood leukocytes, $10^{\wedge} 3 / \mu \mathrm{L}$ & $8.38 \pm 2.63$ & $6.01 \pm 2.47$ & 0.045 \\
\hline Blood neutrocytes, $10^{\wedge} 3 / \mu \mathrm{L}$ & $7.18 \pm 2.30$ & $4.86 \pm 2.23$ & 0.030 \\
\hline Blood lymphocytes, $10^{\wedge} 3 / \mu \mathrm{L}$ & $0.75 \pm 0.46$ & $0.65 \pm 0.49$ & 0.633 \\
\hline Blood CRP, mg/dL & $3.73 \pm 4.12$ & $3.29 \pm 4.05$ & 0.811 \\
\hline CSF glucose, $\mathrm{mg} / \mathrm{dL}$ & $24.8 \pm 10.8$ & $44.7 \pm 16.5$ & 0.005 \\
\hline CSF protein, mg/dL & $234(166-1006)$ & $139(57-428)$ & 0.013 \\
\hline CSF leukocytes, cells $/ \mu \mathrm{L}$ & $107.2(10-658.7)$ & $37.0(3.7-238)$ & 0.020 \\
\hline CSF neutrocytes, cells $/ \mu \mathrm{L}$ & $24.7(0.7-474.7)$ & $5.0(0.4-36)$ & 0.092 \\
\hline CSF lymphocytes, cells/ $\mu \mathrm{L}$ & $79.5(6-259.4)$ & $37.0(2-205)$ & 0.045 \\
\hline CSF ADA, IU/L & $12.0 \pm 4.0$ & $12.4 \pm 7.5$ & 0.916 \\
\hline No (\%) of patients with sequela & $7(70)$ & $4(31)$ & 0.061 \\
\hline
\end{tabular}

Abbreviations: CRP, C reactive protein; CSF, cerebrospinal fluid; $A D A$, adenosine deaminase

The correlation coefficient of each parameter has also been calculated. In patients with BM, correlations were found among CSF leukocytes, neutrocytes, and lymphocytes (leukocytes $v s$ neutrocytes: $\rho=0.99$ ( $p$ <0.001), leukocytes vs lymphocytes: $\rho=0.86(p<0.001)$, neutrocytes vs lymphocytes: $\rho=0.81(p=$ $0.001))$. Moreover, a correlation was found between CSF protein and glucose $(\rho=-0.68(p=0.02))$. However, correlation was not observed among other parameters. In patients with $\mathrm{TM}$, correlations were found between CSF leukocytes and lymphocytes $(\rho=0.91(p<0.001))$, leukocytes and neutrocytes $(\rho=$ $0.68(p<0.001))$, and protein and $\operatorname{ADA}(\rho=0.68(p=0.001))$. Correlation was not observed among other parameters. In particular, correlations could not be found between ADA and CSF cell counts (ADA $v S$ leukocytes: $\rho=0.22(p=0.373)$; ADA vs lymphocytes: $\rho=0.15(p=0.536)$; ADA vs neutrocytes: $\rho=-0.03$ $(p=0.895))$.

\section{Receiver operating characteristics}

The diagnostic value of each CSF or blood test parameter has also been analyzed using AUC.

Figure 2 shows the ROC curves for each parameter in BM. An area of 0.90 (95\% confidence interval (CI): $0.71-1)$ and 0.89 (95\% Cl: 0.67-1) was found for CSF glucose and CSF protein, respectively. No 
discriminant ability was shown in other parameters, including CSF leukocyte count $(0.46$ (95\% Cl: $0.10-$ $0.82)$ ) and its fractionations (neutrocytes: 0.57 (95\% Cl: 0.22-0.92); lymphocytes: 0.59 (95\% Cl: $0.20-$ 0.97)) and blood test parameters (leukocytes: 0.57 (95\% Cl: 0.18-0.96); CRP: 0.54 (95\% Cl: 0.15-0.93)). As for the significance between each parameter, the AUC of CSF leukocytes was lower than that of the CSF protein $(p=0.046)$.

Figure 3 shows the ROC curves for each parameter in TM. Areas of 0.85 ( $95 \% \mathrm{Cl}: 0.69-1), 0.76$ (95\% Cl: $0.56-0.96), 0.77$ (95\% Cl: $0.56-0.97), 0.75$ (95\% Cl: $0.54-0.96), 0.74$ (95\% Cl: $0.52-0.96)$, and 0.78 (95\% Cl: 0.57-0.99) were found for CSF glucose, CSF protein, CSF leukocytes, CSF lymphocytes, blood leukocytes, and blood neutrocytes, respectively. No discriminant ability was shown in other parameters, including CSF neutrocytes (0.68 (95\% Cl: 0.43-0.92)), CSF ADA (0.42 (95\% Cl: $0.15-0.68)$ ), CRP (0.56 (95\% Cl: $0.31-0.81)$ ), and blood lymphocytes (0.54 (95\% Cl: $0.27-0.81)$ ). As for the significance between each parameter, the AUC of the CSF lymphocytes was higher than that of the CSF ADA $(p=0.018)$.

\section{Discussion}

This study suggests that initial CSF analysis predicts the existence of enhancement on contrastenhanced MRI scans of the central nervous system, both in patients with BM and those with TM. In patients with BM, higher CSF protein and lower CSF glucose were associated with the presence of the enhancement on MRI, while CSF leukocyte or neutrocyte counts did not show difference. In contrast, not only CSF protein and glucose but also leukocyte and lymphocyte counts were associated with the enhancement in patients with TM. Furthermore, CSF ADA did not show either correlation with the CSF leukocyte count or the discriminant ability of the MRI findings in patients with TM.

Firstly, CSF analysis just before or right after treatment predicted the presence of enhancement on contrast-enhanced MRI scans, both in patients with BM and those with TM. Despite the suggestion that the enhancement may reflect the severity of the patient especially in $\mathrm{TM}^{8}$, quantitative research about the relationship between CSF and MRI findings has been rare in each disease. Our study implied the correlation of the two tests and also revealed that, while the CSF protein and glucose correlate to the MRI findings in both diseases, the CSF cell count correlate to it only in TM and not in BM.

Secondly, the CSF ADA did not correlate with the CSF leukocyte or lymphocyte counts and also did not show discriminant ability of the existence of the MRI findings in patients with TM. ADA, which is a useful marker of $\mathrm{TM}^{9}$, has been hypothesized to have a crucial role in the differentiation and proliferation of lymphoid cells ${ }^{10}$, and the CSF ADA has been suggested to reflect the activity of T lymphocytes in the CSF rather than their total number ${ }^{11}$. Since the CSF ADA did not correlate with the enhancement on MRI scans, our study result suggests that the CSF lymphocytes, which were higher in those with enhancement, do not play an important role in the BBB breakdown.

Our findings about the CSF cell count and CSF ADA indicate the mechanism of BBB breakdown in infectious meningitis. The pathophysiology of BBB breakdown in BM has been hypothesized to consist 
the following three components: the toxicity of bacteria itself, the cytotoxicity of microglia, and the inflammation caused by lymphocytes recruited into the $\operatorname{CSF}^{12,13,14}$. Our result that CSF cell count in BM could not predict the MRI findings suggests that those recruited lymphocytes are not fully responsible for the breakdown of BBB; moreover, bacteria itself and microglia ${ }^{15}$ are its main contributors. By contrast, the following process has been theorized to be the pathophysiology of TM: after the infection of endothelial cells and the infiltration of infected immune cells, BBB get damaged by the cytotoxicity of microglia; then, uninfected immune cells are recruited through disrupted BBB and worsen the inflammation ${ }^{16}$. Considering our result and hypothesis about the CSF ADA, infected endothelial cells and microglia may be the main contributors to the breakdown of BBB in TM. In addition, CSF cell counts in both diseases may just reflect the passage of time after recruitment of those cells through disrupted BBB. This explains the conflicting results in CSF cell count between the two diseases.

This study has several limitations. First, the hospitals are general hospitals with neurosurgery department. Referred patients may tend to have higher complication rates; therefore, bias existed in this regard. Second, this study included test results obtained at other hospitals, and those lab tests or MRI scans might not be fully comparable.

Now, this study has shown that CSF analysis predicts the presence of enhancement on contrastenhanced MRI scans. Additionally, as discussed so far, the toxicity of pathogens and microglia rather than recruited lymphocytes is assumed to play an important role in BBB dysfunction both in BM and TM. Further study is required to examine the precise sensitivity and specificity of each item as a test standard, with a greater number of patients and using other sequence including contrast-enhanced fluid-attenuated inversion recovery, which is known to have higher detection sensitivity of the effect in infectious meningitis ${ }^{17,18}$.

\section{Conclusions}

CSF analysis predicts the existence of enhancement on contrast-enhanced MRI scans of the central nervous system both in patients with BM and those with TM. The CSF protein and glucose correlate to the MRI findings in both diseases, while the CSF cell count correlate to it only in TM and not in BM. Moreover, CSF ADA did not show the discriminant ability of the MRI findings in patients with TM. Taken all together, our findings about the CSF cell count and CSF ADA indicate the mechanism of the BBB breakdown in BM and TM; bacteria itself, infected endothelial cells, and microglia may be the main contributors to it.

\section{Abbreviations}

ADA: adenosine deaminase

AUC: area under the curve 
BBB: Blood-brain barrier

BM: Bacterial meningitis

CRP: C-reactive protein

CSF: Cerebrospinal fluid

ROC: receiver operating characteristic

TM: tuberculous meningitis

\section{Declarations}

\section{Ethics approval and consent to participate:}

This study was approved by the local institutional review board (the Institutional Review Board for Clinical Research, No. NCGM-G-004149-00) and was performed according to the ethical standards of the 1964 Declaration of Helsinki and its later amendments. Patient consent was not required by our institutional review board because of the retrospective nature of the study and the lack of patient interaction.

\section{Consent for publication:}

All images are entirely unidentifiable and there are no details on individuals reported.

\section{Availability of data and materials:}

The datasets supporting the conclusions of this article are included within the article and its additional file.

\section{Competing interests:}

The authors declare that they have no competing interests.

\section{Funding:}

The authors report no sources of funding for the research.

\section{Authors' contributions:}

RG and NA designed and conceptualized the study. RG played a major role in the acquisition and analysis of data, and also drafted the manuscript. YH, HK, AC, TY, and ST interpreted the data and participated in the manuscript preparation. All authors read and approved the final manuscript.

\section{Acknowledgements:}


Not applicable.

\section{References}

1. Shih RY, Koeller KK. Bacterial, fungal, and parasitic infections of the central nervous system: radiologic-pathologic correlation and historical perspectives. Radiographics. 2015;35:1141-1169.

2. Meltzer CC, Fukui MB, Kanal E, Smirniotopoulos JG. MR imaging of the meninges. Part I. Normal anatomic features and nonneoplastic disease. Radiology. 1996;201:297-308.

3. Smirniotopoulos JG, Murphy FM, Rushing EJ, Rees JH, Schroeder JW. Patterns of contrast enhancement in the brain and meninges. Radiographics. 2007;27:525-552.

4. Thwaites GE, Macmullen-Price J, Chau TTH, Mai PP, Dung NT, Simmons CP, et al. Serial MRI to determine the effect of dexamethasone on the cerebral pathology of tuberculous meningitis: an observational study. Lancet Neurol. 2007;6:230-236.

5. Marais S, Thwaites G, Schoeman JF, Török ME, Misra UK, Prasad K, et al. Tuberculous meningitis: a uniform case definition for use in clinical research. Lancet Infect Dis. 2010;10:803-812.

6. Hanley JA, McNeil BJ. A method of comparing the areas under receiver operating characteristic curves derived from the same cases. Radiology. 1983;148:839-843.

7. Youden WJ. Index for rating diagnostic tests. Cancer. 1950;3:32-35.

8. Verdon R, Chevret S, Laissy JP, Wolff M. Tuberculous meningitis in adults: review of 48 cases. Clin Infect Dis. 1996;22:982-988.

9. Ribera E, Martinez-Vazquez JM, Ocaña I, Segura RM, Pascual C. Activity of adenosine deaminase in cerebrospinal fluid for the diagnosis and follow-up of tuberculous meningitis in adults. J Infect Dis. 1987; 155:603-607.

10. Piras MA, Gakis C, Budroni M, Andreoni G. Adenosine deaminase activity in pleural effusions: an aid to differential diagnosis. Br Med J. 1978;2:1751-1752.

11. Choi SH, Kim YS, Bae IG, Chung JW, Lee MS, Kang JM, et al. The possible role of cerebrospinal fluid adenosine deaminase activity in the diagnosis of tuberculous meningitis in adults. Clin Neurol Neurosurg. 2002;104:10-15.

12. Barichello T, Generoso JS, Simões LR, Goularte JA, Petronilho F, Saigal P, et al. Role of microglial activation in the pathophysiology of bacterial meningitis. Mol Neurobiol. 2016;53:1770-1781.

13. Koedel U, Klein M, Pfister HW. New understandings on the pathophysiology of bacterial meningitis. Curr Opin Infect Dis. 2010;23:217-223.

14. Mook-Kanamori BB, Geldhoff M, van der Poll T, van de Beek D. Pathogenesis and pathophysiology of pneumococcal meningitis. Clin Microbiol Rev. 2011;24:557-591.

15. Sharief MK, Ciardi M, Thompson EJ. Blood-brain barrier damage in patients with bacterial meningitis: association with tumor necrosis factor-alpha but not interleukin-1 beta. J Infect Dis. 1992;166:350358. 
16. Wilkinson RJ, Rohlwink U, Misra UK, van Crevel R, Mai NTH, Dooley KE, et al. Tuberculous Meningitis International Research Consortium. Tuberculous meningitis. Nat Rev Neurol. 2017;13:581-598.

17. Splendiani A, Puglielli E, Amicis RD, Necozione S, Masciocchi C, Gallucci M. Contrast-enhanced FLAIR in the early diagnosis of infectious meningitis. Neuroradiology. 2005;47:591-598.

18. Alonso A, Eisele P, Ebert AD, et al. Leptomeningeal contrast enhancement and blood-CSF barrier dysfunction in aseptic meningitis. Neurol Neuroimmunol Neuroinflamm. 2015;2:e164.

\section{Figures}

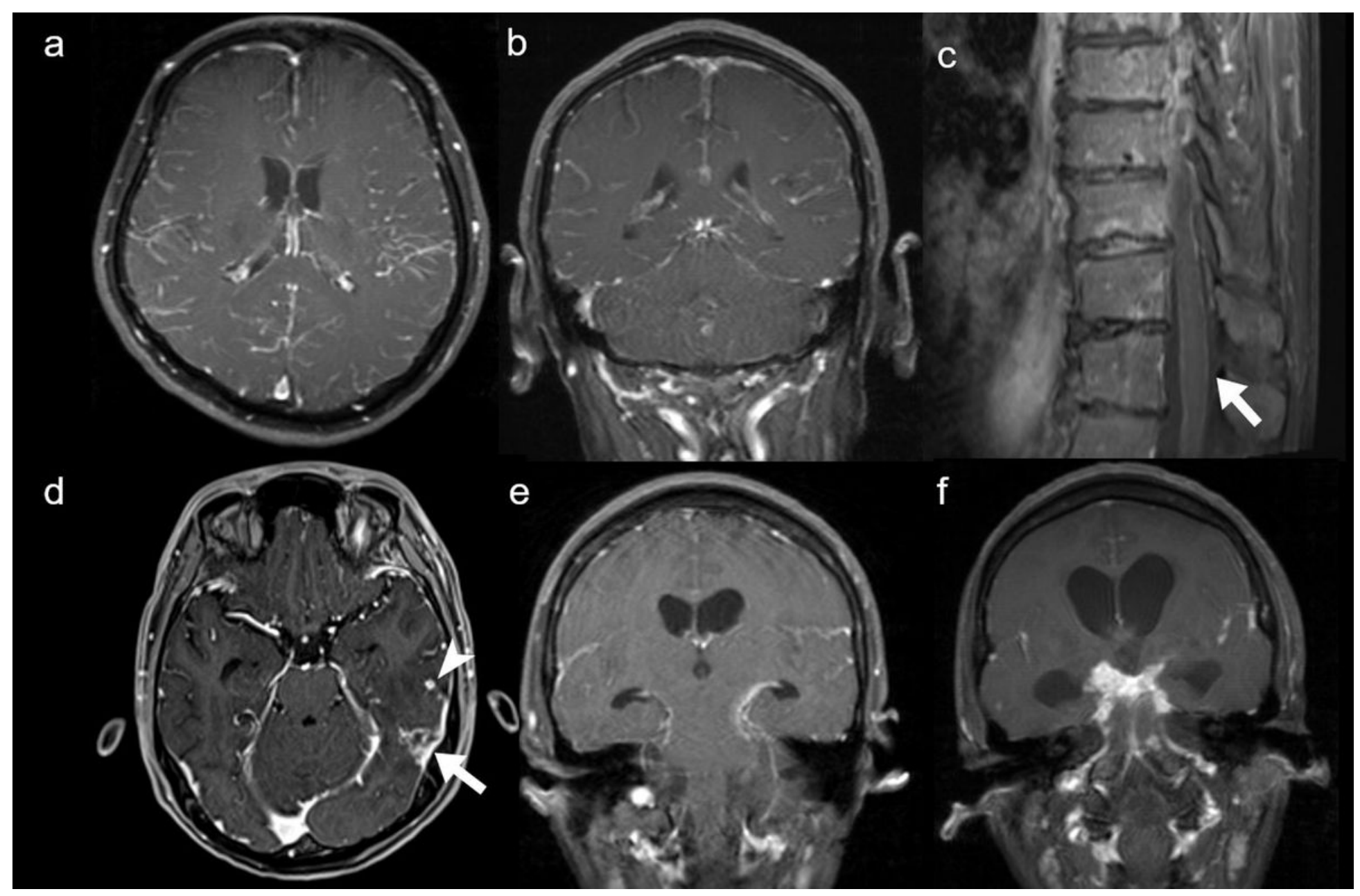

Figure 1

Exemplary MRI of patients with contrast enhancement on T1-weighted MRI (a) Head MRI of a 58-year-old patient with bacterial meningitis (BM). The scan shows leptomeningeal and dural enhancement. On the coronal plane of the same scan (b), the enhancement is widely seen on the surface and the sulcus of both the cerebrum and cerebellum. (c) Spinal MRI of a 71-year-old patient with BM. Enhancement of the meninges is seen in the lumbar spinal cord (arrow). The patient also had a complication of pyogenic spondylitis. (d) Head MRI of an 18-year-old patient with tuberculous meningitis (TM). The scan shows enhancement of the meninges (arrow) in addition to a tuberculoma (arrowhead) in the left temporal lobe. 
(e) Head MRI of a 36-year-old patient with TM. The scan shows enhancement of the meninges, especially on the left ambient cistern. The case developed hydrocephalus, which is shown in the image taken three months later ( $f)$.

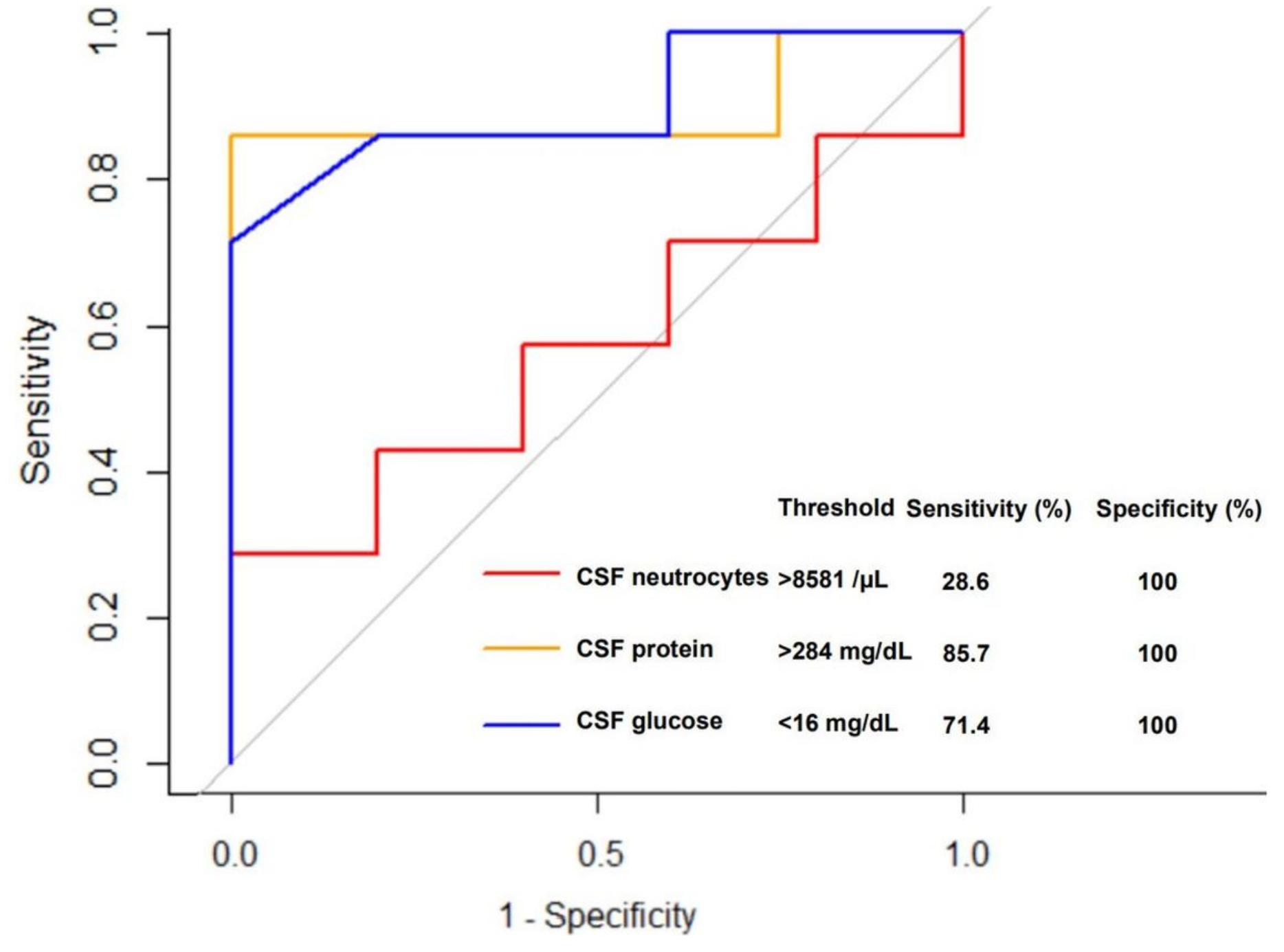

\section{Figure 2}

Sensitivity and false-positive rate (1-specificity) for receiver operating characteristic for each parameter in bacterial meningitis The sensitivity and specificity of each parameter as a predictor of enhancement on MRI are shown in the inset table. Although the sample size is small, the CSF protein exhibits the highest sensitivity and specificity, while the CSF neutrocyte count is hardly useful as a predictor of the enhancement on MRI. Statistical analysis proved that the AUC of the CSF neutrocytes was significantly lower than that of the CSF protein. AUC, area under the curve 


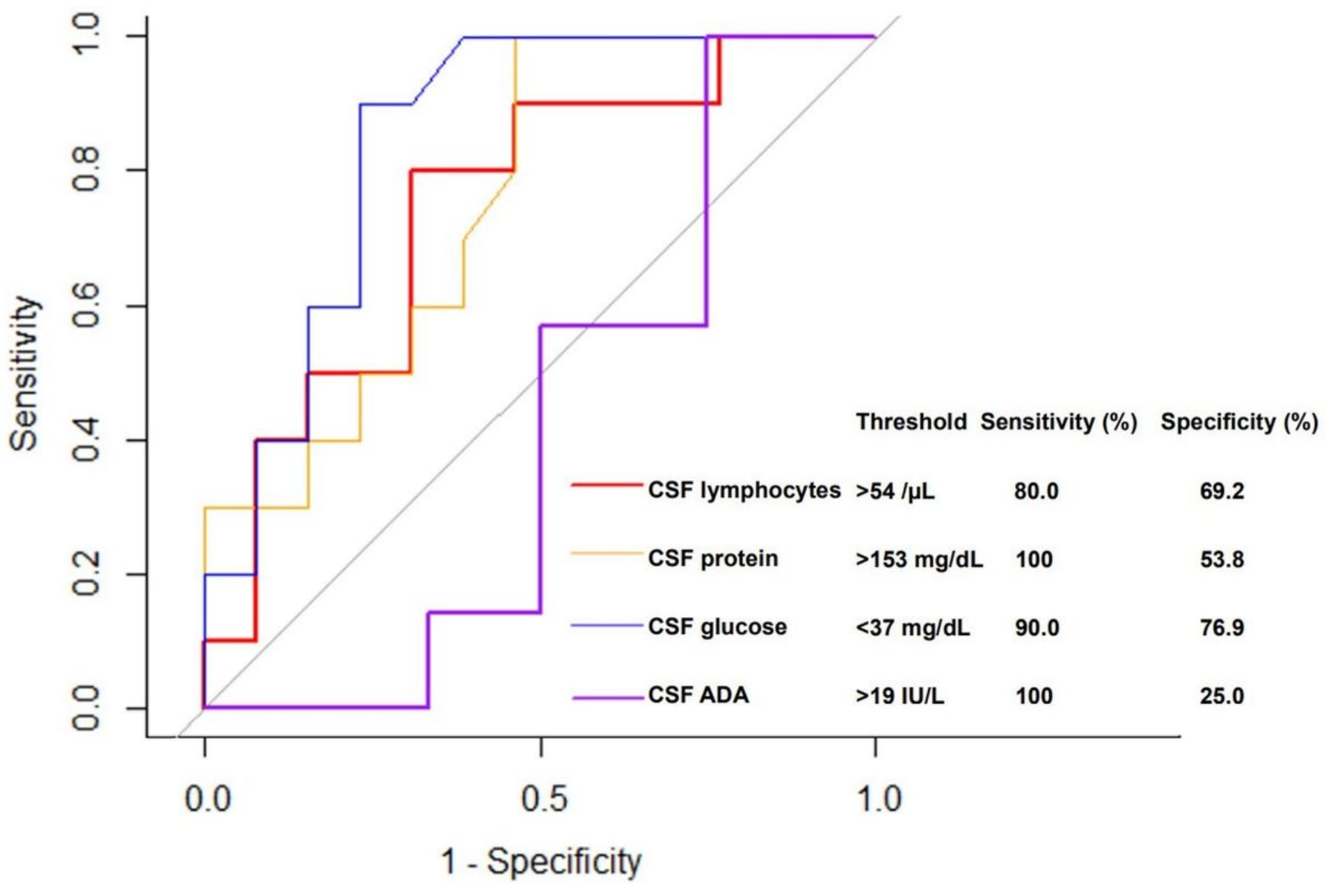

Figure 3

Sensitivity and false-positive rate (1-specificity) for receiver operating characteristic for each parameter in tuberculous meningitis The sensitivity and specificity of each parameter as a predictor of enhancement on MRI are shown in the inset table. The CSF glucose exhibits the highest sensitivity and specificity, as well as CSF lymphocytes. The specificity of the CSF protein is relatively low, while the CSF ADA is hardly useful as a predictor of the enhancement on MRI. Statistical analysis supports the idea, as the AUC of lymphocytes was significantly higher than that of ADA. ADA, adenosine deaminase; AUC, area under the curve

\section{Supplementary Files}

This is a list of supplementary files associated with this preprint. Click to download.

- AdditionalFile1.xIsx 